\title{
Different soliton pulse order effects on the fiber communication systems performance evaluation
}

\author{
Mahmoud M. A. Eid ${ }^{1}$, Abd El-Naser A. Mohammed ${ }^{2}$, Ahmed Nabih Zaki Rashed ${ }^{3}$ \\ ${ }^{1}$ Department of Electrical Engineering, College of Engineering, Taif University, Kingdom of Saudi Arabia \\ ${ }^{2}$ Higher Institute of Electronic Engineering, Bilbies, 10th of Ramadan, Egypt \\ ${ }^{3}$ Electronics and Electrical Communications Engineering Department, Faculty of Electronic Engineering, \\ Menoufia University, Egypt
}

\begin{tabular}{|c|c|}
\hline Article Info & ABSTRACT \\
\hline Article history: & \multirow{9}{*}{$\begin{array}{l}\text { The study outlined the soliton pulse order effects on the performance } \\
\text { efficiency of the optical transceiver systems. The power after fiber is } \\
\text { reported for various Soliton pulse order. Max optical signal power (SP) and } \\
\text { min optical noise power (NP) are clarified versus time after optical fiber for } \\
\text { various soliton pulse order. As well as the max electrical power amplitude } \\
\text { against time period is demonstrated after electrical filter for various soliton } \\
\text { pulse order. It is reported that the optical transceiver performance efficiency } \\
\text { can be upgraded with the first soliton order pulse. The soliton technique is } \\
\text { used for high speed communication transmission systems. Soliton technique } \\
\text { is used to compensate the dispersion and balanced with nonlinear effects. } \\
\text { The soliton order effects is then discussed to choose the suitable soliton order } \\
\text { for high speed system performance efficiency. The soliton techniques can be } \\
\text { used also for extended ultra high transmion distance with high data rates. }\end{array}$} \\
\hline Received Sep 21, 2020 & \\
\hline Revised Jun 13, 2021 & \\
\hline Accepted Jul 14, 2021 & \\
\hline Keywords: & \\
\hline Butterworth optical filter & \\
\hline Gaussian pulse & \\
\hline Laser rate equations & \\
\hline Soliton order & \\
\hline
\end{tabular}

This is an open access article under the CC BY-SA license.

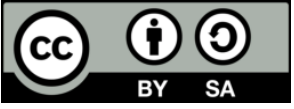

Corresponding Author:

Ahmed Nabih Zaki Rashed

Electronics and Electrical Communications Engineering Department

Faculty of Electronic Engineering

Menoufia University, Egypt

E-mail: ahmed.rashed@el-eng.menofia.edu.eg

\section{INTRODUCTION}

The most problem in optical communication systems is the increase need for high bit rates and max fiber reach with high transmission quality [1]-[6]. The increase capacity in optical fibers lines that are already used, there are many techniques for this purpose. The increase in transmitted channels number by using ultra dense wavelength division multiplexing schemes [7]-[15]. So it is possible to use one optical fiber line to carry maximum transmitted channels up to 100 channels and each channel acts optical wavelength different from each other [16]-[24].

There is a development in optical fiber lines that reach to the stage to establish optical networks [25]-[29]. These optical networks that include all optical components. These networks can be classified into passive or active linear or nonlinear optical networks. The suitable transmission distance in passive optical networks is between 10 to $20 \mathrm{~km}$ between sender and receiver. The suitable transmission distance in active optical networks is 80 to $90 \mathrm{~km}$. linear/nonlinear passive optical communication networks can be modified its distance by using all optical amplifiers [30]-[35].

Fiber Bragg grating (FBG) employed with dispersion compensation over different transmission distances for NRZ at $20 \mathrm{~Gb} / \mathrm{s}$ [36]-[39]. It was revealed that the signal quality slightly changed over longer transmission distance when using FBG [12]-[20]. So, FBG is preferred especially for longer fiber lengths. 
The effects of applying different input power levels on the system performance in case of using FBG as a dispersion compensator have been studied in detail [40]-[43]. It was found that as the power level increases, the fiber nonlinearity effects are also increasing. Wavelength division multiplexing (WDM) system has been modeled using cascaded fiber Bragg grating. The system performance has been analyzed for two cases; with using the cascaded FBG and without using the cascaded FBG. A significant improvement in system performance has been achieved when using cascaded FBG [44]-[55].

The Soliton pulse order effects on the performance efficiency of the optical transceiver systems is studied and simulated. The power after fiber is reported for various Soliton pulse order. Max optical signal power and min optical noise power are clarified versus time after optical fiber for various Soliton pulse order. As well as the max electrical power amplitude against time period is demonstrated after electrical filter for various Soliton pulse order. It is reported that the optical transceiver performance efficiency can be upgraded with the first soliton order pulse. FBG device compensates dispersion in the fiber line and the dispersion can be controlled.

\section{RESEARCH METHOD}

Figure 1 has outlined the Soliton pulse order effects on the optical transceiver systems. The data source generator generates stream of bits sequence. The sequence of bits is reconfigured through the Gaussian pulse generators. Soliton pulses with various orders can be generated with the gaussian pulse generator. The laser rate equations are responsible for the generation of $1550 \mathrm{~nm}$ wavelength and a power of $10 \mathrm{dBm}$. LiNb mach zehnder modulators are responsible for modulated the electro-optic signal. The sig. is injected into $300 \mathrm{~km}$ distance. Sig. is degraded with the cable and attenuations due to the losses. The signal can be processed through the butterworth optical filter. The losses in the signal can be treated through $5 \mathrm{~m}$ length EDFA and Raman amplifiers. The optical signal can be converted to electrical signal and can be treated from the ripples through PIN Photodetector and low pass butterworth electrical filter respectively. 3R regenerator can be retimed/reshaped the signal again.

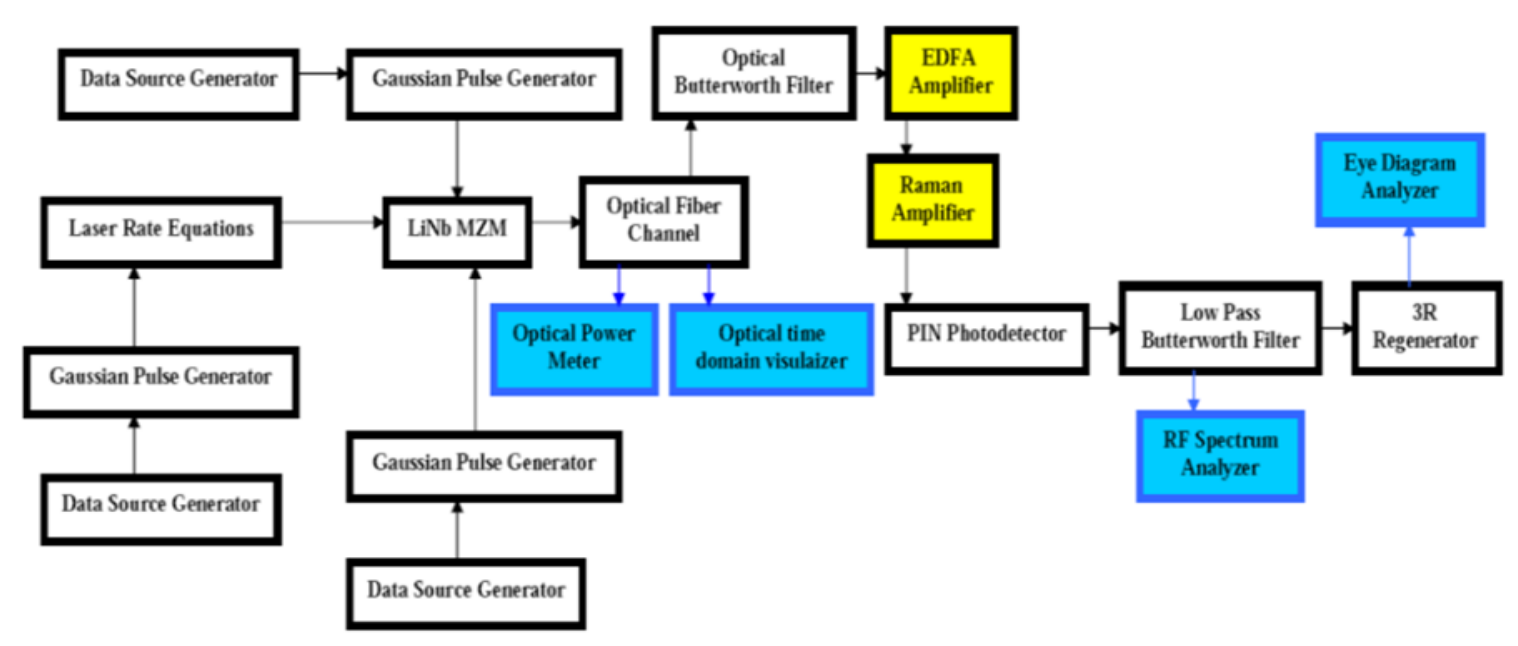

Figure 1. Proposed simulation model description

Max Q, min BER are tested by BER analyzer. The nonlinear pulse propagation in optical fiber can be estimated by [4],

$$
n(I)=n_{0}+n_{2} I
$$

with linear index $\left(\mathrm{n}_{0}\right)$, I is the optical intensity, and nonlinear index $\left(\mathrm{n}_{2}\right)$. The chirped hyper-secant Soliton pulse can be modeled by [4],

$$
u(\xi, \tau)=N_{s} \operatorname{Sech}(p t) \exp [i K \xi-i q \ln (\operatorname{Cosh}(p \tau))]
$$

where the parameters $\xi$, $\tau$, and $\mathrm{u}$ are estimated by [4]: 


$$
\begin{aligned}
& \xi=\frac{z\left|\beta_{2}\right|}{T_{0}^{2}} \\
& \tau=\frac{T}{T_{0}} \\
& u=\sqrt{\frac{\gamma T_{0}^{2}}{\left|\beta_{2}\right|}}
\end{aligned}
$$

with $\beta_{2}$ is the propagation constant, $\mathrm{T}_{0}$ is the bit period, and $\mathrm{z}$ is Soliton propagation distance.

\section{RESULTS AND DISCUSSION}

The Soliton pulse order effects with the engagement between EDFA/Raman amplifiers on the performance efficiency of the transceiver systems have been simulated. We have demonstrated the total optical power after optical fiber for various soliton order pulse. The max optical SP and optical NP with time after optical fiber for various soliton order pulse are reported. As well as the max electrical power amplitude versus periodic time after electrical filter for the first soliton order pulse is clarified. The clarified results depend on the items in Table 1.

Table 1. List of items in this proposed work [3], [5], [9]

\begin{tabular}{lc}
\hline \multicolumn{1}{c}{ Parameters } & Values/Units \\
\hline Laser rate equation source & \\
Wavelength & $1550 \mathrm{~nm}$ \\
Power & $10 \mathrm{dBm}$ \\
Drive current & $38 \mathrm{~mA}$ \\
Mod. Peak current & $28 \mathrm{~mA}$ \\
Line width & $10 \mathrm{MHz}$ \\
LiNb MZM modulator & $5 \mathrm{~dB}$ \\
Insertion loss & $20 \mathrm{~dB}$ \\
Extinction ratio & \\
Optical link & \\
Length & $3000 \mathrm{~km}$ \\
Wavelength & $1550 \mathrm{~nm}$ \\
Loss & $0.14 \mathrm{~dB} / \mathrm{km}$ \\
EDFA/Raman Amplifiers & \\
EDFA Length & $5 \mathrm{~m}$ \\
Core radius & $2.2 \mu \mathrm{m}$ \\
PIN Photodetector & \\
Responsitivity & $1 \mathrm{~A} / \mathrm{W}$ \\
Gain & $5 \mathrm{~dB}$ \\
\hline
\end{tabular}

Figure 2(a) clarifies the total optical power after optical fiber for the first soliton order pulse. The total optical power is $83.366 \mu \mathrm{W}(10.79 \mathrm{dBm})$ with the first soliton order pulse. Figure 2(b) demonstrates the total optical power after optical fiber for the second soliton order pulse. The total optical power is $45.64 \mu \mathrm{W}$ $(13.406 \mathrm{dBm})$ with the second soliton order pulse. Figure 2(c) illustrates the total optical power after optical fiber for the third soliton order pulse. The total optical power is $39.429 \mu \mathrm{W}(14.401 \mathrm{dBm})$ with the third soliton order pulse. With the first soliton order pulse the total optical power can be enhanced than other high Soliton pulse order.

The max optical SP and min optical NP versus time after optical fiber for the first soliton order pulse outlined in Figure 3(a). The optical power is $0.000312 \mathrm{~W}$ and $\min \mathrm{NP}$ is $-1.486 \times 10^{-5} \mathrm{~W}$ with the first Soliton pulse order. The max optical SP and min optical NP versus time after optical fiber for the second soliton order pulse are clarified in Figure 3(b). The peak SP is $0.00024299 \mathrm{~W}$ and min NP is-1.1571 x $10^{-5} \mathrm{~W}$ with the second Soliton pulse order. The max optical SP and min optical NP versus time after optical fiber for the third soliton order pulse are demonstrated in Figure 3(c). Optical power is $0.00020829 \mathrm{~W}$ and min NP is $9.9184 \times 10^{-6} \mathrm{~W}$ with the third soliton pulse order. The max optical SP can be upgraded and min optical NP can be reduced by using the first soliton order pulse. 


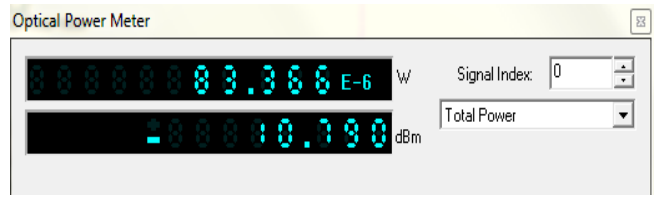

(a)

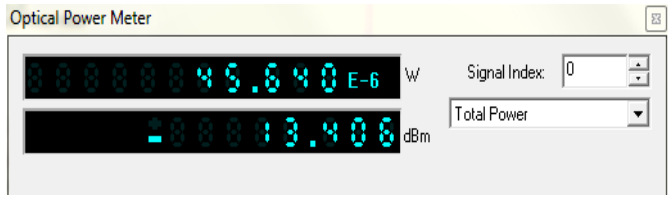

(b)

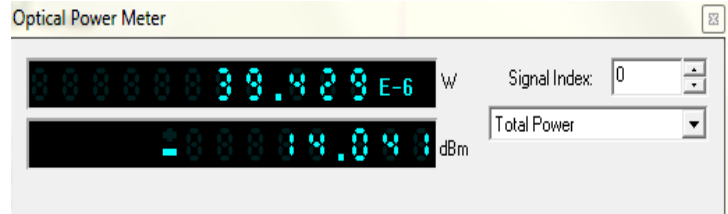

(c)

Figure 2. These figures are; (a) total power after fiber for the first soliton order pulse; (b) total power after fiber for the second soliton order pulse; (c) total power after fiber for the third soliton order pulse

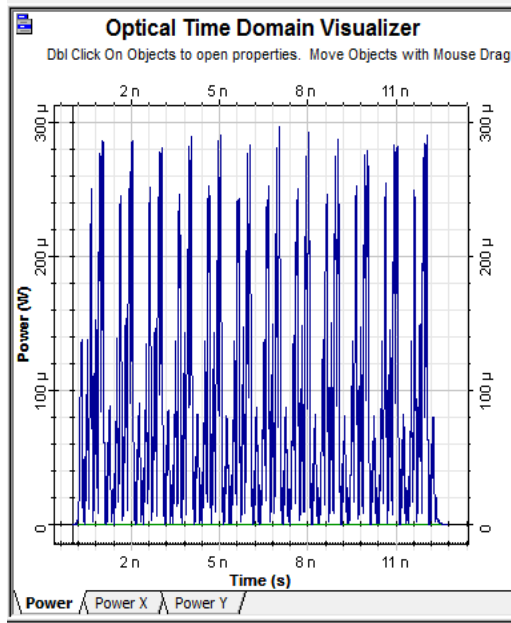

(a)

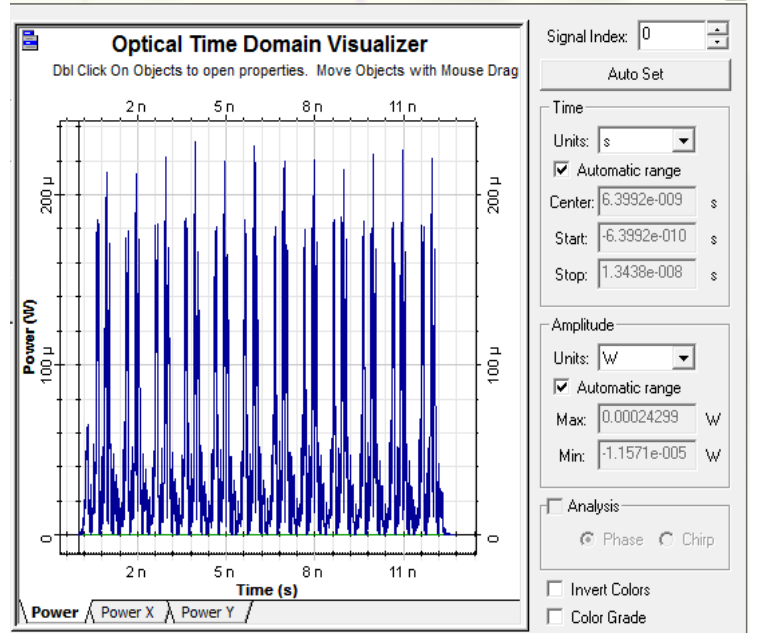

(b)

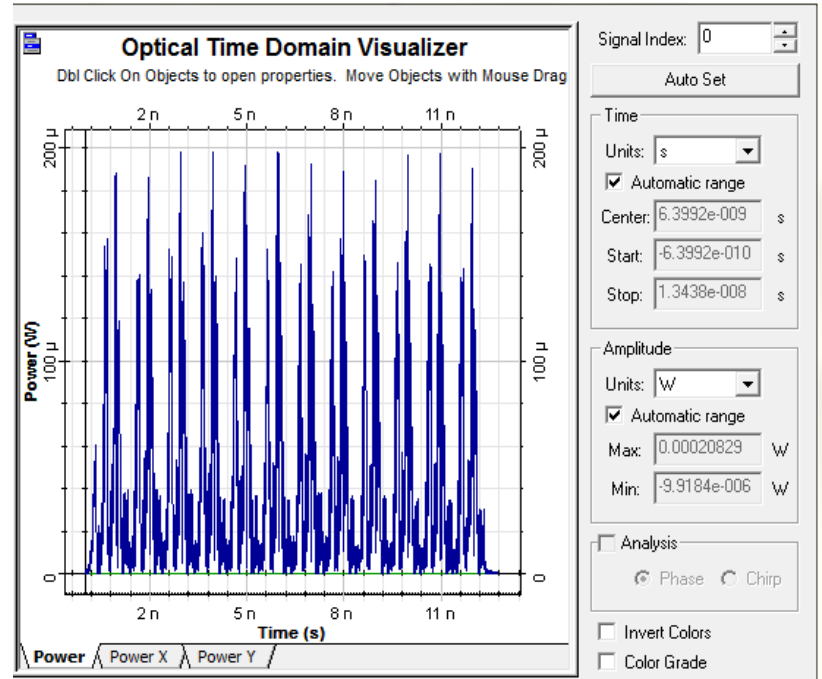

(c)

Figure 3. These figures are; (a) Max optical signal and min optical NPs versus time after optical fiber for the first soliton order pulse, (b) Max optical signal and min optical NPs versus time after optical fiber for the second soliton order pulse; (c) Max optical signal and min optical NPs versus time after optical fiber for the third soliton order pulse 
The max electrical SP and min electrical NP against frequency after electrical filter for the first soliton order pulse are clarified in Figure 4(a). Electrical power is $0.656534 \mathrm{dBm}$ and min electrical SP is $104.793 \mathrm{dBm}$ with the first soliton order pulse. The max electrical SP and min electrical NP against frequency after electrical filter for the second soliton order pulse are reported in Figure 4(b). The peak SP is $0.881793 \mathrm{dBm}$ and min electrical SP is $-104.72 \mathrm{dBm}$ with the second soliton order pulse. The max electrical SP and min electrical NP against frequency after electrical filter for the third soliton order pulse are outlined in Figure 4(c). Electrical power is $-1.57224 \mathrm{dBm}$ and min electrical SP is $-104.687 \mathrm{dBm}$ with the third soliton order pulse. The max electrical SP can be upgraded and the min electrical NP can be reduced in the presence of first soliton order pulse. The max electrical power amplitude versus time after electrical filter for the first soliton order pulse is shown in Figure 5(a). The Q is 9.1376 and BER is $3 \times 10^{-20}$ with the first Soliton pulse order. The max electrical power amplitude versus time after electrical filter for the second soliton order pulse is indicated in Figure 5(b). The Q is 7.30714 and BER is $1.27335 \times 10^{-13}$ with the second soliton pulse order. The max electrical power amplitude versus time after electrical filter for the third soliton order pulse is reported in Figure 5(c). The Q is 5.87183 and BER is $1.9 \times 10^{-9}$ with the third soliton pulse order. The Q factor can be enhanced and BER can be reduced by using the first soliton order pulse.

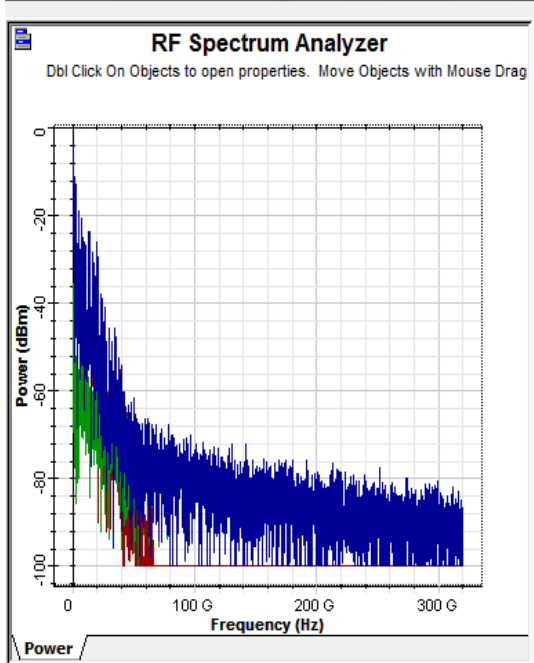

(a)

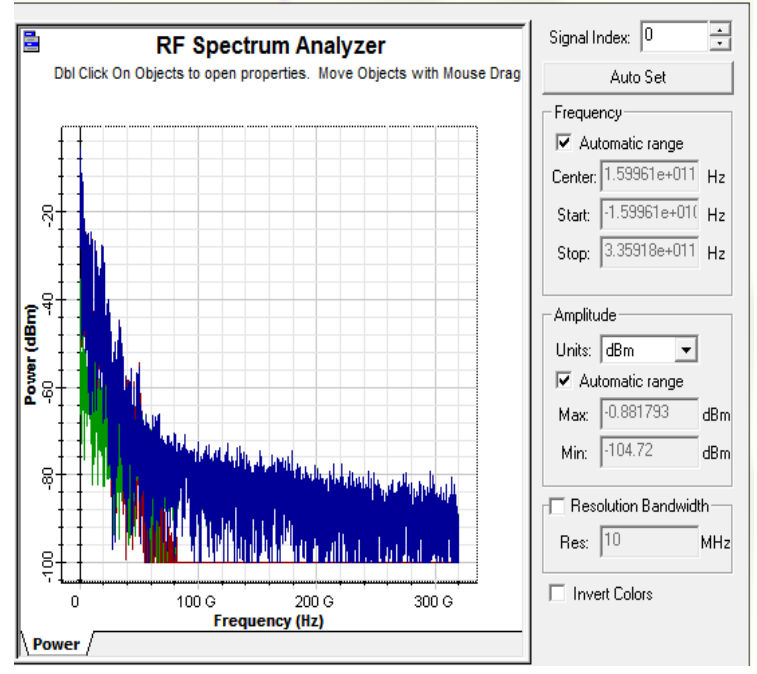

(b)
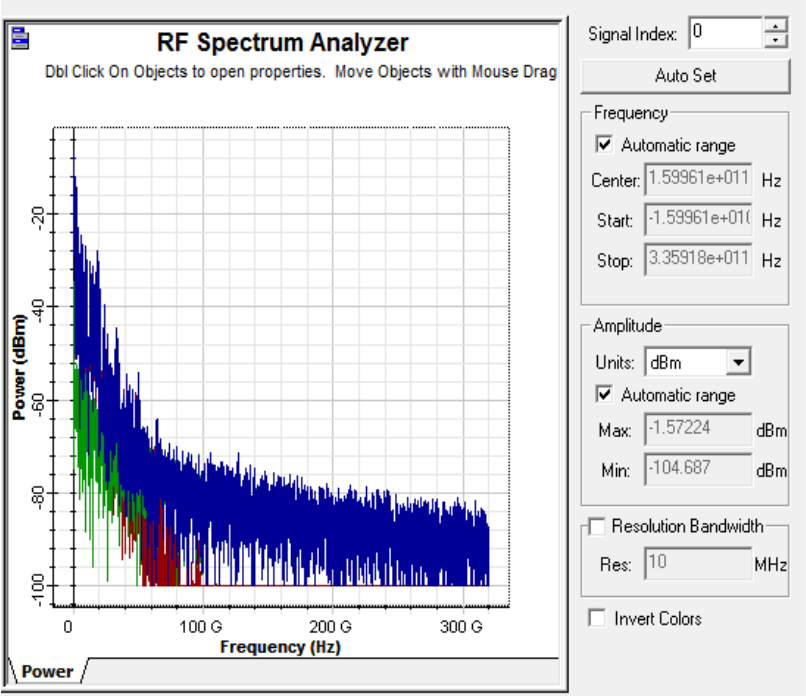

(c)

Figure 4. These figures are; (a) Max electrical SP and min electrical NP against frequency after electrical filter for the first soliton order pulse; (b) Max electrical SP and min electrical NP against frequency after electrical filter for the second soliton order pulse; (c) Max electrical SP and min electrical NP against frequency after electrical filter for the third soliton order pulse 


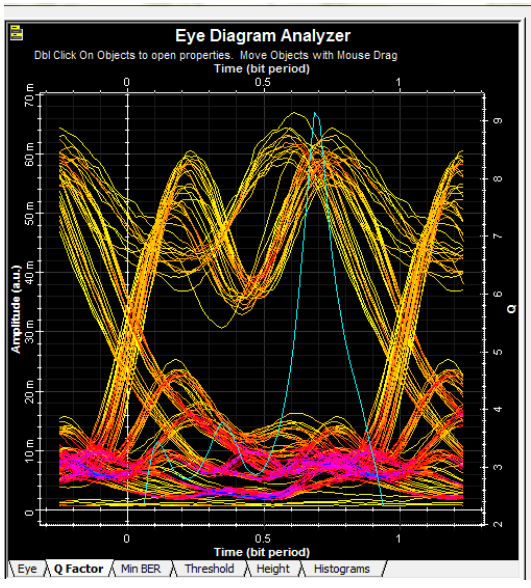

(a)

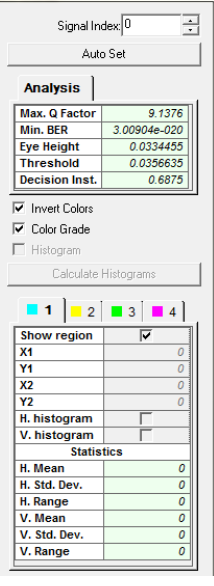

(c)

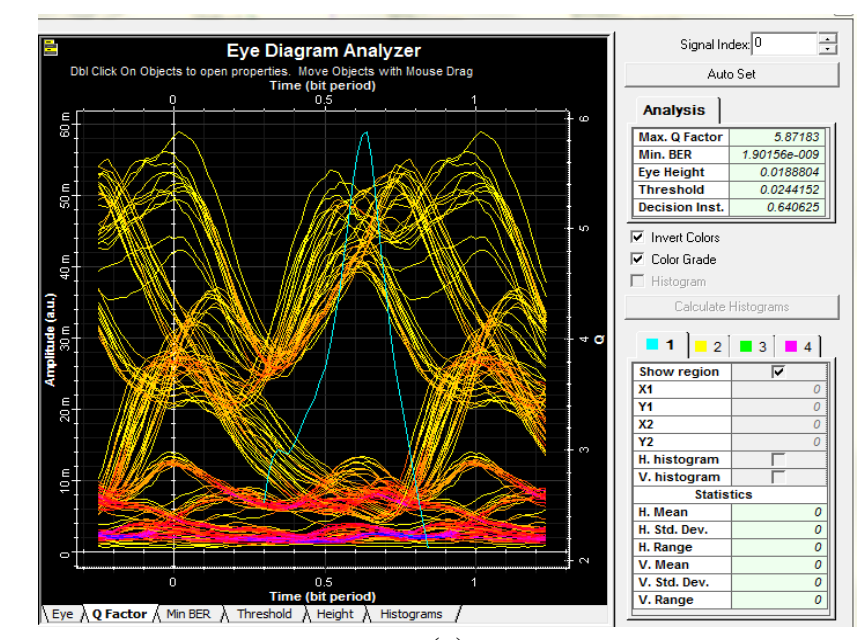

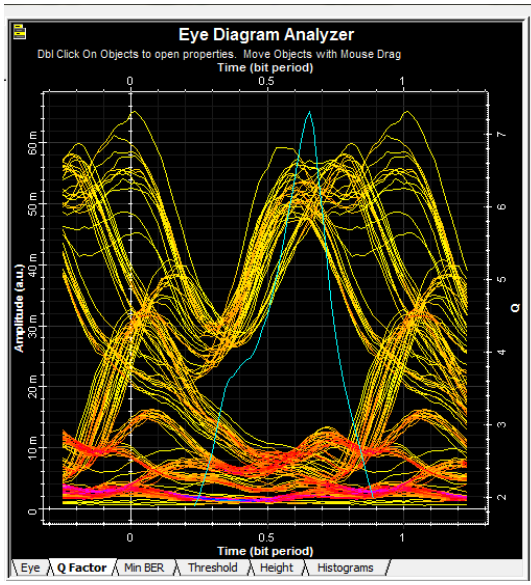

(b)

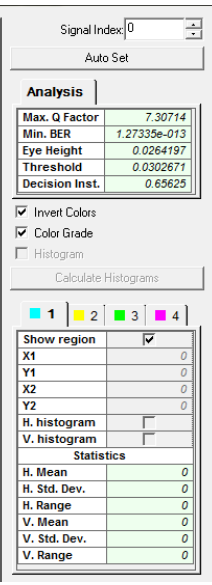

Figure 5. These figures are; (a) Max electrical power amplitude versus time after electrical filter for the first soliton order pulse; (b) Max electrical power amplitude versus time after electrical filter for the second soliton order pulse;

(c) Max electrical power amplitude versus time after electrical filter for the third soliton order pulse

\section{CONCLUSIONS}

We have been simulated the Soliton pulse order variation effects on the performance efficiency of the transceiver communication systems. It is clarified that total optical power, max optical/electrical SPs and Q factor decrease linearly with the increase of the Soliton pulse order. It is outlined that the Q factor, max optical/electrical SPs and total optical power can be enhanced and min BER can be reduced by using the first soliton order pulse. Max Q factor can be enhanced with the choice of suitable propagation reach and amplification technique. The study emphasized that the important role of both EDFA/Raman amplifiers for strength the signal and upgrading Max Q values.

\section{REFERENCES}

[1] U. Brinkmann, "Nonlinear optics-Spatial solitons of moderate power interact," Laser Focus World, vol. 40, no. 4, pp. 32-48, 2004.

[2] I. Maimistov, "Solitons in nonlinear optics," Quantum Electron., vol. 40, no. 9, pp. 756-781, 2010, doi: 10.1070/QE2010v040n09ABEH014396.

[3] X. G. Lin, W. J. Liu, and M. Lei, “Oscillating solitons in nonlinear optics," Pramana, vol. 86, no. 3, pp. 575-580, 2016, doi: 10.1007/s12043-015-1020-x.

[4] Y. Song, Xujie Shi, Chengfa Wu, Dingyuan Tang and Han Zhang, "Recent progress of study on optical solitons in fiber lasers," Appl. Phys. Rev., vol. 6, paper id: 021313, 2019; doi: 10.1063/1.5091811.

[5] Y. D. Cui and X. M. Liu, "Graphene and nanotube mode-locked fiber laser emitting dissipative and conventional solitons," Opt. Express, vol. 21, no. 16, pp. 18969-18974, 2013, doi: 10.1364/OE.21.018969. 
[6] Jiemei Wang, Chunhui Lu, Shangbin Li and Zhengyuan Xu, "100 m/500 Mbps Underwater Optical Wireless Communication Using an NRZ-OOK Modulated 520nm Laser Diode," Optics Express, vol. 27, no. 9, pp. 1217112181, 29 Apr. 2019, doi: 10.1364/OE.27.012171.

[7] Bikash Kumar Paul, Sujan Chakma, Md. Abdul Khalek, and Kawsar Ahmed, "Silicon Nano Crystal Filled Ellipse Core Based Quasi Photonic Crystal Fiber with Birefringence and Very High Nonlinearity," Chinese Journal of Physics, Elsevier, vol. 56, no. 6, pp. 2782-2788, December 2018, doi: 10.1016/j.cjph.2018.09.030.

[8] Hosam Abd Elrazek Mohamed Ali, El-Sayed Soliman A. Said, and Mohamed Ebrhim Yousef, "Effect of Environmental Parameters on The Performance of Optical Wireless Communication," Int. J. of Optics, vol. 2019, pp. 1-12, 2019, doi: 10.1155/2019/1828275.

[9] A. Nandhini, K. Gokulakrishnan, "Design of Ultra High Capacity DWDM System with Different Modulation Formats," Int. J. of Sci. Eng. and Res. (IJSER), vol. 3, no. 5, pp. 41-45, May 2015.

[10] R. Bin Talib, "Multi Slot Amplitude Coding Technique for High Speed Optical Fiber Communication System," Faculty of Electrical and Electronic Engineering, University Tun Hussein Onn Malaysia, Ph.D. Thesis, June 2015.

[11] Swatantra Kumar Tiwari, Prof A. K. Jaiswal, Mukesh Kumar, Manvendr, Satyesh Sharan Singh, "Performance Analysis of Optical Amplifiers for Incorporation in Optical Network," Int. J. of Adv. Tech. in Eng. and Sci. (IJATES), vol. 2, no. 10, pp. 238-247, Oct. 2014.

[12] M. Syuhaimi Ab-Rahman, Majid Moghaddasi, "A Comparison Between Electrical and Optical Chromatic Dispersion Compensation in Wavelength Division Multiplexing Network Regarding to Electrical Pulse Shapes," $J$. of Comp. Science, vol. 8, no. 1, pp. 76-83, 2012, doi: 10.3844/jcssp.2012.76.83.

[13] Andre G. Metzger, "An Electronic Equalization Technique for Nonlinear Dispersion Compensation in Optical Fiber Communications Links,” University of California, San Diego, Ph.D. Thesis, 2011.

[14] Bentahar Attaouia and Kandouci Malika, "Optimized of Erbium-Doped-Fiber Amplifiers (EDFA) Parameters in Hybrid Passive Optical Network (WDM/TDM-PON) Systems with 512 Users," World Applied Sciences Journal, vol. 33, no. 6, pp. 1042-1051, 2015, doi: 10.5829/idosi.wasj.2015.33.06.272.

[15] Akchita Khemariya, Aditi Agrawal, A. K. Jaiswal, "WDM EDFA+RFA Hybrid Amplifier for Gain Equalization in C Band," International Journal of Computer Applications, vol. 128, no. 16, pp. 22-25, Oct. 2015, doi: 10.5120/ijca2015906748.

[16] Hardeep Singh, Sukhwinder Singh, "Performance Analysis of Hybrid Optical Amplifiers in 120 x 10 Gbps WDM Optical Network with Channel Spacing of $50 \mathrm{GHz}$," The Second Int. Conf. on Advances In Computing, Communication and Information Technology, USA, ISBN: 978-1-63248-051-4, 2014, pp. 55-58, doi: 10.15224/ 978-1-63248-051-4-27

[17] Lekshmi. S. R, Sindhu. N, "Spectral Response of FWM in EDFA for Long-Haul Optical Communication," Int. J. of Innovative Research in Computer and Communication Engineering (IJIRCCE), vol. 2, no. 10, pp. 6091-6095, October 2014.

[18] Anuja Dhokar, S. D. Deshmukh, "Overview of EDFA for the Efficient Performance Analysis," IOSR Journal of Engineering (IOSRJEN), International organization of Scientific Research, vol. 04, no. 03, pp. 1-8, March 2014, doi: $10.9790 / 3021-04340108$.

[19] Parveen Bagga, Himali Sarangal, "Analysis of DWDM System with Hybrid Amplifiers at Different Transmission Distance," Int. J. of Research in Electronics and Computer Engineering (IJRECE), vol. 3, no. 1, pp. 64-66, January - March 2015.

[20] Chinky Rani, Kulwinder Singh and Bhawna Utreja, "Performance Analysis of Bi-Directional Broadband Passive Optical Network Using Travelling Wave Semiconductor Optical Amplifier," Int. J. of Eng. Research and Applications (IJERA), vol. 3, no. 4, pp. 114-118, July-August 2013.

[21] Frederic Laforce, "Low Noise Optical Receiver Using Si APD," Society of Photo-Optical Instrumentation Engineers (SPIE), vol. 7212, no. 10, 2009, doi: 10.1117/12.809071.

[22] Mukesh Kumar, Sandeep Singh, Jay Singh, Rohini Saxena, "Performance Analysis of WDM/SCM System Using EDFA," International Journal of Advanced Research in Computer Science and Software Engineering (IJARCSSE), vol. 2, no. 6, pp. 112-116, June 2012.

[23] Malti, Meenakshi Sharma, Anu Sheetal, "Comparison of CSRZ, DRZ and MDRZ Modulation Formats for High Bit Rate WDM-PON System Using AWG," Int. J. of Emerging Technology and Advanced Engineering (IJETAE), vol. 2, no. 6, pp. 83-87, June 2012.

[24] Md. Shazzad Hossain, Sujan Howlader, Rinku Basak, "Investigating the Q-Factor and BER of a WDM System in Optical Fiber Communication Network by Using SOA," Int. J. of Innovation and Scientific Research, vol. 13, no. 1, pp. 315-322, January 2015.

[25] Mostafa Zaman Chowdhury, Md. Shahjalal, Md. Khalid Hasan, and Yeong Min Jang, "The Role of Optical Wireless Communication Technologies in 5G/6G and IoT Solutions: Prospects, Directions, and Challenges," Applied Science, vol. 9, no. 20, October 2019. doi: 10.3390/app9204367.

[26] Marek S. Wartak, "Computational Photonics, An Introduction with MATLAB," Cambridge University Press, ISBN 978-1-107-00552-5, New York, USA, 2013.

[27] Prachi Sharma, Rohit Kumar Arora, Suraj Pardeshi and Mandeep Singh, "Fibre Optic Communications: An Overview," Int. J. of Emerging Technology and Advanced Engineering (IJETAE), vol. 3, no. 5, pp. 474-479, May 2013.

[28] Mijanur Rahim, Abdul Touhid Bar, Anjumanara Begam and Md Asraful Sekh, "Investigation of Dispersion Compensation Methods for the Data Rates of 2.5 and 10 Gbps Using Standard and Dispersion Compensated Fibers," Journal of Emerging Technologies and Innovative Research (JETIR), vol. 6, no. 5, pp. 731-734, May 2019, doi: 10.6084/m9.figshare.jetircj06153.

[29] Bostjan Batagelj, Vijay Janyani, and Saso Tomazic, "Research Challenges in Optical Communications towards 2020 and Beyond," J. of Microelectronics, Electronic Components and Materials, vol. 44, no. 3, pp. 177-184, Sep. 2014. 
[30] Kimberly Kolb, "Signal-to-Noise Ratio of Geiger-Mode Avalanche Photodiode Single-Photon Counting Detectors," Optical Engineering, vol. 53, no. 8, pp. 1-8, August 2014, doi: 10.1117/1.OE.53.8.081904.

[31] Meng Peun Tan, "Improving the Efficiency and Threshold Current of Photonic Crystal Vertical-Cavity SurfaceEmitting Lasers," Master Thesis, University of Illinois at Urbana-Champaign, Urbana, Illinois, 2009.

[32] Adnan Affandi, and Othman AL-Rusaini, "Implementation of Fiber Optic Communication System Using Developed Computer Program," IOSR J. of Electrical and Electronics Eng. (IOSR - JEEE), vol. 11, no. 3, pp. 122142, May-June 2016.

[33] Sayeeda Khatoon, A. K. Jaiswal, and Aditi Agrawal, "Performance Evaluation of Post and Symmetrical DCF Technique with EDFA in $32 \times 10,32 \times 20$, and $32 \times 40$ Gbps WDM Systems," International Journal of Current Engineering and Technology (IJCET), vol. 7, no. 4, pp. 1416-1421, August 2017.

[34] Sabina, and Manpreet Kaur, "Performance Comparison of Pre-, Post-, and Symmetrical Dispersion Compensation for $96 \times 40 \mathrm{~Gb} / \mathrm{s}$ DWDM System Using DCF," International Journal of Application or Innovation in Engineering and Management (IJAIEM), vol. 6, no. 7, pp. 235-244, July 2017.

[35] Petar Spalevic, Dejan Milic, Branimir Jaksic, Mile Petrovic, and Ilija Temelkovski, "Simulation Influence of the Thermal Noise of PIN Photodetector on Performance DWDM Optical Network," XLVII Int. Scientific Conf. on Information, Comm. and Energy Systems and Tech., Sofia, vol. 2, 28-30 June 2012, pp. 315-318, doi: 10.1515/joc-2019-0152.

[36] Alex Mutig, "High Speed VCSELs for Optical Interconnects," Ph.D. Dissertation, Berlin University, Springer Theses, 2010.

[37] N. K. Kahlon, and G. Kaur, "Various Dispersion Compensation Techniques for Optical System: A Survey," Open J. of Communications and Software, vol. 1, no. 1 pp. 64-73, May 2014, doi: 10.15764/CS.2014.01006.

[38] S. M. Jahangir Alam, M. Rabiul Alam, Guoqing Hu, and Md. Zakirul Mehrab, "Bit Error Rate Optimization in Fiber Optic Communications," International Journal of Machine Learning and Computing, vol. 1, no. 5, pp. 435440, December 2011, doi: 10.7763/IJMLC.2011.V1.65.

[39] Wissam Mahjoob Osman, Khalid Hammed Billal and Amin Babiker Al Nabi, "Bit Error Rate Performance for Optical Fiber System,” Journal of Electrical and Electronic Systems, vol. 7, no. 1, 1000250, pp. 1-13, 2018, doi: 10.4172/2332-0796.1000250.

[40] Dandan Zhu and Colin J. Humphreys, "Solid-State Lighting Based on Light Emitting Diode Technology," Optics in Our Time, pp. 87-118, 2016, doi: 10.1007/978-3-319-31903-2_5.

[41] Ansheng Liu, Ling Liao, Doron Rubin, Hat Nguyen, Berkehan Ciftcioglu, Yoel Chetrit, Nahum Izhaky, and Mario Paniccia, "High-Speed Optical Modulation Based on Carrier Depletion in a Silicon Waveguide," Opt. Express, vol. 2, pp. 660-668, 2007, doi: 10.1364/OE.15.000660.

[42] R. S. Rifia Reen, G. Ravi, "Performance Evaluation of BER and Q Factor for EDFA Based WDM Network," International Journal of Engineering Research and Technology (IJERT), vol. 3, no. 16, pp. 1-5, 2015.

[43] Xiaoyue Wu, "Simulation Study of Epitaxial Regrown Vertical-Cavity Surface Emitting Lasers," Maser thesis, Royal Institute of Technology, Stockholm, Sweden, 2011.

[44] Meng Peun Tan, Ansas M. Kasten, Joshua D. Sulkin, and Kent D. Choquette, "Planar Photonic Crystal VerticalCavity Surface-Emitting Lasers," IEEE Journal of Selected Topics in Quantum Electronics, vol. 19, no. 4, July/August 2013, doi: 10.1109/JSTQE.2013.2241398.

[45] Hideki Ohe, Hiromasa Shimizu, and Yoshiaki Nakano, "InGaAlAs Multiple-Quantum Well Optical Phase Modulators Based on Carrier Depletion,” IEEE Photon. Technology Letters, vol. 19, no. 22, pp. 1816-1818, November 15, 2007, doi: 10.1109/LPT.2007.907566.

[46] Neha and R. L Sharma, "Performance Analysis of 8-Channel WDM System Using Symmetrical DCF and Optigrating," International Journal of New Trends in Electronics and Communication (IJNTEC), vol. 2, no. 4, June 2014.

[47] Aashima Bhardwaj, Gaurav Soni, "Performance Analysis of 20Gbps Optical Transmission System Using Fiber Bragg Grating," International J. of Scientific and Research Publications (IJSRP), vol. 5, no. 1, pp. 1-4, January 2015.

[48] Petar Spalevic, Dejan Milic, Branimir Jaksic, Mile Petrovic, and Ilija Temelkovski, "Simulation influence of the thermal noise of PIN photodetector on performance DWDM optical network," XLVII Int. Scientific Conf. on Information, Comm. and Energy Systems and Tech., ICEST 2012, Sofia, vol. 2, 28-30 June 2012, pp. 315-318.

[49] Kaushal Kumar, A. K. Jaiswal, Mukesh Kumar and Nilesh Agrawal, "Performance Analysis of Dispersion Compensation Using Fiber Bragg Grating (FBG) in Optical Communication," Int. Journal of Current Engineering and Technology (IJCET), vol. 4, no. 3, pp. 1527-1531, June 2014.

[50] Qiaoyin Lu, Weihua Guo, Diarmuid C. Byrne, and John F. Donegan, "Design of Low V-pi High-Speed GaAs Travelling-Wave Electro-Optic Phase Modulators Using an n-i-p-n Structure, ” IEEE Photonics Technology Letters, vol. 20, pp. 1805-1807, 2008, doi: 10.1109/lpt.2008.2005009.

[51] Tomasz Czyszanowski, Maciej Dems, Robert P. Sarzala, Kassimir Panajotov, and Kent D. Choquette, "Photonic Crystal VCSELs: Detailed Comparison of Experimental and Theoretical Spectral Characteristics," IEEE Journal of Selected Topics in Quantum Electronics, vol. 19, no. 5, October 2013, doi: 10.1109/JSTQE.2013.2247568.

[52] Farah Z. Jasim, Khalid Omar, and Zainuriah Hassan, "Temperature Effect on VCSEL Output Performance," Optoelectronics and Advanced Materials-Rapid Communications, vol. 3, no. 11, 2010.

[53] José Mulet Pol, "Semiconductor Laser Dynamics, Compound-Cavity, Polarization and Transverse Modes," Ph. D., France, 2012.

[54] Julien Macario, Peng Yao, Rownak Shireen, Christopher A. Schuetz, Shouyuan Y. Shi, and Dennis W. Prather, "Development of Electro-Optic Phase Modulator for 94 GHz Imaging System," Journal of Lightwave Technology, vol. 27, pp. 5698 - 5703, 2009, doi: 10.1109/JLT.2009.2035641.

[55] Mazin Al Noor, "Green Radio Communication Networks Applying Radio-Over-Fiber Technology for Wireless Access,” Ph. D. Dissertation, Middlesex University, United Kingdom, December 2011. 